\title{
Axonal excitability and conduction alterations caused by levobupivacaine in rat
}

\author{
SEÇKIN TUNCER ${ }^{*}$ \\ TÜLAY TUNCER PEKER ${ }^{2}$ \\ ILLKSEN BURAT ${ }^{1}$ \\ ERHAN KIZILTAN ${ }^{3}$ \\ BARKIN İLHAN ${ }^{1}$ \\ NIZAMETTIN DALKILIÇ 1 \\ ${ }^{1}$ N.E. University, Meram Faculty \\ of Medicine, Biophysics Department \\ Konya, Turkey \\ ${ }^{2}$ Ankara University, Faculty \\ of Medicine, Anesthesiology \\ Department, Ankara, Turkey \\ ${ }^{3}$ BaşkentUniversity, Faculty \\ of Medicine, Physiology Department \\ Ankara, Turkey
}

Accepted May 10, 2017

Published online June 14, 2017

\begin{abstract}
In this study, effects of the long-acting amide-type local anesthetic levobupivacaine on axonal conduction and excitability parameters of the rat sciatic nerve were thoroughly examined both in vitro and in vivo. In order to deduce its effects on isolated nerve conduction, compound nerve action potential (CNAP) recordings were performed using the suction method over sciatic nerves of Wistar rats before and after administration of $0.05 \%$ (1.7 mmol $\mathrm{L}^{-1}$ ) levobupivacaine. Levobupivacaine caused complete CNAP area and amplitude depression by blocking conduction in a timedependent manner.

To assess the influence of levobupivacaine on in vivo excitability properties, threshold-tracking (TT) protocols were performed at sciatic nerves of rats injected with perineural $0.05 \%$ (1.7 mmol $\mathrm{L}^{-1}$ ) levobupivacaine or vehicle alone. Charge-duration TT results revealed that levobupivacaine increases the rheobase and decreases the strength-duration time constant, suggesting interference of the anesthetic with the opening of $\mathrm{Na}^{+}$channels. Twenty and $40 \%$ threshold electrotonus curves were found for both groups to follow the same paths, suggesting no significant effect of levobupivacaine on $\mathrm{K}^{+}$channels for either the fastest or relatively slow conducting fibers. Current-threshold relationship results revealed no significant effect on axonal rectifying channels. However, according to the results of the recovery cycle protocol yielding the pattern of excitability changes following the impulse, potential deviation was found in the recovery characteristics of $\mathrm{Na}^{+}$channels from the absolute refractory period. Consequently, conduction blockage caused by levobupivacaine may not be due to the passive (capacitive) properties of axon or the conductance of potassium channels but to the decrease in sodium channel conductance.
\end{abstract}

Keywords: levobupivacaine, nerve conduction, excitability, threshold electrotonus

Levobupivacaine is a long-acting amide-type local anesthetic that interferes with voltage-gatedion channels. The anesthetic and/or analgesic effects of levobupivacaine have been shown to be largely similar, however less toxic compared to bupivacaine at the same

*Correspondence; e-mail: tuncerseckin@gmail.com 
dose, in many comparative studies, including those of epidural, peripheral nerve blockage, local infiltration and peribulbar administration (1). The sensory block due to levobupivacaine is longer than that of bupivacaine when used on peripheral nerves, not imposing any cardiovascular or CNS toxicity because of its enantio selectivity. On the contrary, bupivacaine has been shown to produce aQTc interval prolongation on ECG and overall depression of EEG signals. Levobupivacaine is a local anesthetic widely known for its blocking effect on $\mathrm{Na}^{+}$channels, but in recent studies it has been shown to affect $\mathrm{K}^{+}, \mathrm{Ca}^{2+}$, transient receptor potential vanilloid 1 (TRPV1) channels and $N$-methyl- $D$-aspartate (NMDA), $\alpha$-amino-3-hydroxy-5-methyl-4-izoxazolepropionic acid (AMPA), and GTP-binding protein coupling receptors (2). Combined effects of levobupivacaine on these ion channels and receptors could result in complicated changes in peripheral nerve conduction and excitability.

Since the ingenious works of Gasser and co-workers in the late 1930s (3), methods depending on the compound nerve action potential (CNAP) recording have been used as a useful means for the evaluation of nerves both for the purpose of research and clinical diagnosis. To get detailed information on the individual activity of nerve fiber groups, CNAP recording from isolated nerves is still considered as the primary method of choice (4). However, thanks to the threshold tracking (TT) method developed by Bostock et al. (5) in the last decade, more parameters about nerve excitability could be acquired reliably. Since the excitability of an axon could be defined as the current required to yield a specific level of response, excitability can be indirectly measured over the applied amount of electrical current. Depolarization of an axon increases its excitability and there by decreases the strength of threshold current needed to cause excitation; hence, a weaker current would be needed to produce the same magnitude of response, and vice versa. On the other hand, hyperpolarization of the axon increases the amount of current needed to produce a comparable response. In this method, a given response amplitude of the target compound muscle action potential (CMAP) or compound nerve action potential (CNAP), consisting of the sum of individual activities of muscle or nerve fibers, is preset. The threshold current strength is then automatically adjusted to elicit this constant target response. These automatically driven patterns of "percent change threshold" are taken to be an accurate representation of axonal excitability under the point of stimulation (6).

A myelinated axon has ion channels, pumps and exchangers responsible for determining axonal excitability. In the node of Ranvier, transient $\mathrm{Na}^{+}$channels $\left(\mathrm{Na}_{\mathrm{t}}\right)$ are densely clustered together with persistent $\mathrm{Na}^{+}$channels $\left(\mathrm{Na}_{\mathrm{p}}\right)$ and slow $\mathrm{K}^{+}$channels $\left(\mathrm{K}_{\mathrm{s}}\right)$, which as a whole contribute to the excitability and resting membrane potential. In addition to $\mathrm{K}_{\mathrm{s}}$ the internodal region of the axon includes fast $\mathrm{K}^{+}$channels $\left(\mathrm{K}_{\mathrm{f}}\right)$ scattered densely, acting to limit re-excitation of the node just after an action potential, and hyperpolarization-activated (inward rectifying) $\mathrm{K}^{+}$channels $(6,7)$.

Levobupivacaine is the frequently preferred local anesthetic, which has previously been shown to be capable of blocking rat sciatic nerve conduction (8). Its effects on nerve conduction and excitability parameters have been extensively studied, using traditional CNAP recording and TT methods, for the appropriate dose of perineural administration. TT is a relatively new method that provides information about the changes in ion channel activity; it enables on going assessment for clinical validation of several studies. Its main advantage over other methods is that it provides, non-invasively, a means for in vivo measurement of ion channel activity (6). The aim of the present study was to investigate in 
detail the effects of levobupivacaine on nerve excitability in order to enlighten the changes in ionic channel activity. In addition, the conduction parameters acquired from the isolated nerve were evaluated for the conduction blockage effect of anesthetic agents by means of the traditional CNAP recording method.

\section{EXPERIMENTAL}

\section{Animals}

The experiments were carried out on 24 male adult Wistar albino rats, weighing 250$350 \mathrm{~g}$, purchased from KONUDAM (N. E. University Experimental Medicine and Application Center, Konya, Turkey). All experimental procedures on animals were carried out in accordance with the ARRIVE guidelines and instructions of the Meram Medical Faculty Experimental Ethics Committee (N. E. University, Konya, Turkey). After birth, rats were housed five per cage at ambient temperature and humidity under a 12/12 h light/dark cycle. All animals received food and water ad libitum. Rats were maintained without any signs of neuro behavioral impairment throughout the experiments. Animals were separated into two groups, i.e., 8 rats for in vitro CNAP recordings from isolated sciatic nerves and 16 rats for in vivo multiple excitability measurements.

\section{CNAP recording}

In order to perform CNAP recording, right sciatic nerves of the first group of rats were dissected at the hind paw after being sacrificed by cervical dislocation under deep anesthesia (intraperitoneal injection of $90 \mathrm{mg} \mathrm{kg}^{-1} \mathrm{ketamine}$ and $10 \mathrm{mg} \mathrm{kg}^{-1}$ xylazine combination). Nerves were then rapidly transferred to the recording chamber super fused with modified Krebs solution (composed of, in mmol L-1: $\mathrm{NaCl} 119, \mathrm{KCl} 4.8, \mathrm{CaCl}_{2} 1.8, \mathrm{MgSO}_{4}$ $1.2, \mathrm{KH}_{2} \mathrm{PO}_{4} 1.2, \mathrm{NaHCO}_{3} 20$, and glucose 10, $\mathrm{pH} 7.4$, and gassed with a mixture of $95 \% \mathrm{O}_{2}$ and $5 \% \mathrm{CO}_{2}$ ) at a constant rate of $5 \mathrm{~mL} \mathrm{~min}^{-1}$ at constant temperature $\left(37 \pm 0.5{ }^{\circ} \mathrm{C}\right)$. Temperature was maintained using a heat jacket in the course of recording experiments. Stimuli were given with a stimulator (Model S88K, Grass Instruments Co., USA) at proximal ends of the nerve trunk via a stimulus isolation unit (Model SIU5, Grass Instruments Co.). Square-wave pulses of supramaximal intensity ( $100 \mu$ s duration and $1 \mathrm{~Hz}$ frequency) were used for nerve stimulation. CNAP recordings were performed via a suction electrode placed at the tibial branch of the isolated nerve trunk. Amplified CNAP signals (Model CP511 AC amplifier, Grass Instruments Co.) were digitized using an A/D converter card (Model PCL 1710, Advantech, Taiwan) at 40 KSPS (kilosamples s ${ }^{-1}$ ) using the open-source CNAP recording software RETICAP (http://icon.unrlabs.org/projects/reticap/) produced in our laboratory and stored on hard disk for further analysis. Initial CNAP recordings without levobupivacaine were considered as the baseline $\left(0^{\text {th }}\right.$-min signals) and referred to as CNAP0. After completing these initial recordings, by substituting the solution with another Krebs solution containing $0.05 \%\left(1.7 \mathrm{mmol} \mathrm{L}^{-1}\right)$ levobupivacaine, CNAP recordings were performed every $5 \mathrm{~min}$ for $30 \mathrm{~min}$ until CNAPs vanished completely. These recordings after the $5^{\text {th }}, 10^{\text {th }}, 15^{\text {th }}, 20^{\text {th }}$ and $25^{\text {th }}$-min are, respectively, referred to as CNAP5, CNAP10, CNAP15, CNAP20 and CNAP25 throughout the text. 


\section{Multiple excitability measurements}

Multiple excitability measurement tests were performed on 16 rats divided into two groups having equal numbers of animals $(n=8)$. Rats in the first group, labeled LEV, were unilaterally administered a percutaneous injection of $0.5 \mathrm{~mL}$ of levobupivacaine $(0.05 \%$, $1.7 \mathrm{mmol} \mathrm{L}^{-1}$ ) into the perineural space below the clear fascia covering the nerve itself, proximal to the bifurcation of the sciatic nerve. The second group (CON) served as a control and received $0.5 \mathrm{~mL}$ of saline $(0.9 \% \mathrm{NaCl})$, which served as a vehicle for levobupivacaine. Before perineural injection, rats were anaesthetized with an intraperitoneal injection of 90 $\mathrm{mg} \mathrm{kg}^{-1}$ ketamine and $10 \mathrm{mg} \mathrm{kg}^{-1}$ xylazine combination to produce deep anesthesia. To maintain the level of anesthesia, supplementary doses were administered as required.

To conduct TT experimental protocols, thirty minutes after the perineural injection of levobupivacaine, the lower back regions of rats were shaved and placed prone on a heated table (MAY RTC9404-A Animal Rectal Temperature Controller, Commat Ltd., Turkey), which was controlled by a rectal thermistor probe to keep the body temperature at $37^{\circ} \mathrm{C}$. A stainless steel stimulating surface electrode pair was placed on the sciatic nerve, and $\mathrm{Ag} /$ $\mathrm{AgCl}$ recording surface electrodes were positioned properly on the gastrocnemius muscle groups innervated by these nerves, very close to the point of perineural injection. The ring electrode serving as ground was attached to the tail. Compound muscle action potentials (CMAPs) were amplified and filtered $(1 \mathrm{~Hz}$ to $3 \mathrm{kHz}$, Warner Instrument DP311 Amplifier, USA) and line interference was removed using an online noise eliminator (HumBug 50/60 $\mathrm{Hz}$ Noise Eliminator, Digitimer Co., UK). The resultant signals were then digitized using a computer having an analog-digital converter at a sampling rate of 10 KSPS (National Instrument NI USB-6251 M series DAQ Device, USA). Stimulation was controlled by QTRACS software connected to a data acquisition unit and a stimulator (DS5 type, Digitimer Co., UK). Fig. 1 illustrates the placement of stimulating and recording electrode pairs and the system used for the TT experiment.

In order to assess multiple excitability parameters, standard TT methods were applied (9). Experimental procedure was performed using the software in which multiple excitability protocol TRONDNF of QTRACS was implemented (H. Bostock, UCL Institute of Neurology, London, UK). Methodology and details of the equipment employed in this procedure were previously described elsewhere $(6,9-12)$. Briefly, the first step in our experiments was to obtain the CMAP of maximal amplitude by gradually increasing the stimulus strength by $2 \%$-steps. TRONDNF protocol mainly depends on the tracking of the current required to generate a target response of $40 \%$ of maximal CMAP amplitude. The stimulus-response, strength-duration relationship, threshold electrotonus, current-threshold relationship and recovery cycle parameters were investigated during the multiple excitability testing procedure.

In the course of electrotonus, excitability was tested during and after polarizing currents of $100 \mathrm{~ms}$ duration, for both \pm 20 and $\pm 40 \%$ of the control threshold current. For the current (mA)-threshold (V) relationship, excitability was tested in the period following 200-ms lasting current pulses for the range of +50 to $-100 \%$ of the control threshold current by steps of $10 \%$. For the recovery cycle, excitability was tested for 18 intervals from 1.5 to $200 \mathrm{~ms}$ following a supramaximal conditioning stimulus. 


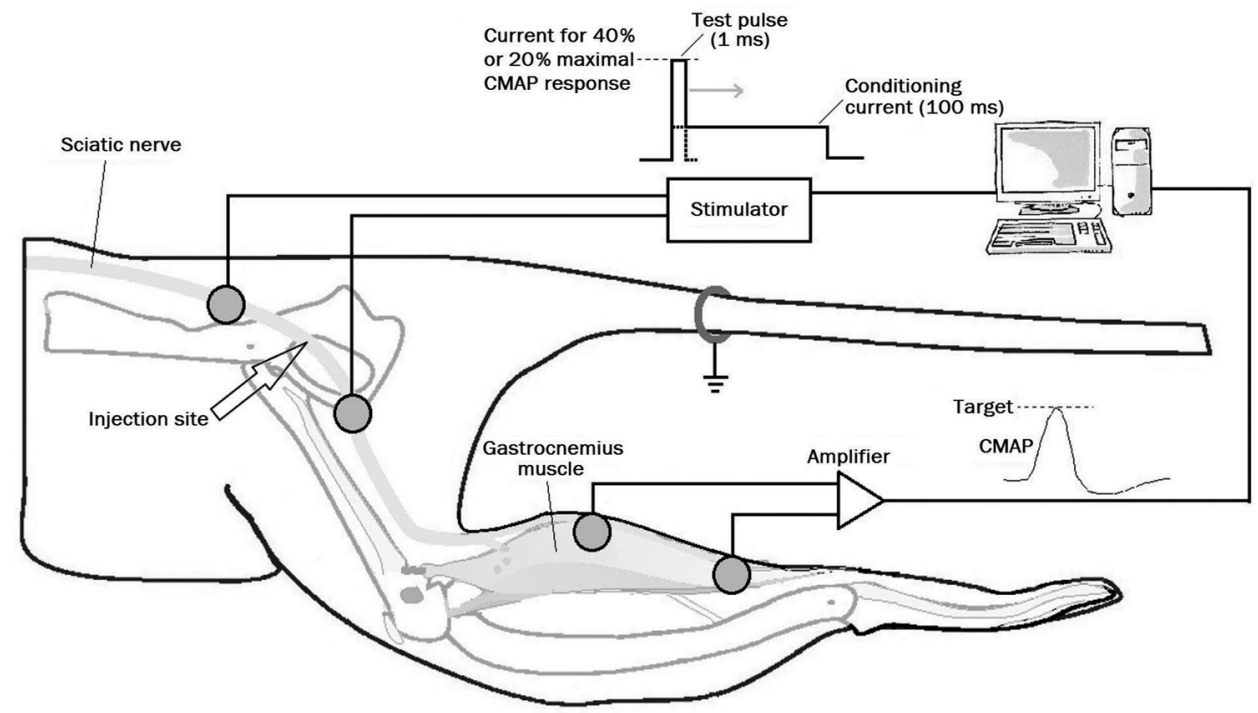

Fig. 1. Schematic diagram of the placement of stimulating and recording electrodes, and the system used for in vivo TT recordings.

\section{Data analysis}

CNAP recordings. - Calculations were conducted on the isolated nerve for two separate conduction velocity $\left(\mathrm{m} \mathrm{s}^{-1}\right)$ values, representing the fastest and the relatively slow conducting groups. The first $\left(\mathrm{CV}_{\text {init }}\right)$ was derived using latencyl $\left(\Delta t_{\text {onset }}\right)$, i.e., the time delay between the instant of stimulus delivery and the associated CNAP onset, while the second $\left(\mathrm{CV}_{\text {peak }}\right)$ was computed for latency $2\left(\Delta t_{\text {peak }}\right)$, the time delay between the stimulus delivery instant and the associated CNAP reaching its maximum value. The distance of the traveling wave used in calculations was $30 \mathrm{~mm}$. The amplitude of CNAPs $(\mathrm{mV})$ and the areas under CNAPs (mV.ms) were also measured.

Multiple excitability tests. - Recordings obtained via the TRONDNF protocol were analyzed and plotted using the QTRACP program, which performs an automated analysis of multiple excitability parameters. Analyses of excitability measurements were mainly done using data obtained from the stimulus-response relationship, strength-duration relationship, threshold electrotonus, current-threshold relationship and recovery cycle protocols, which are the sub-protocols of TRONDNF, as previously described elsewhere (8).

Statistics. - Unless otherwise specified, comparisons for time dependent effects of levobupivacaine were made using the one-way analysis of variance (ANOVA), followed by Duncan's post-hoc test for multiple comparisons where ANOVA yielded a significant result. Student's $t$-test was used to determine whether levobupivacaine did or did not affect excitability parameters. Reliability of our results was tested using Cohen's $d$ effect size calculation method and according to the obtained $d$ results for all parameters, sample size was 
found to be sufficient (13). Statistical analyses were performed using the GraphPad Prism 5 software, where $p$-values of $<0.05$ were regarded as significant. Data were presented as mean \pm SEM throughout the text.

\section{RESULTS AND DISCUSSION}

\section{CNAP parameters}

The CNAP depression effect of levobupivacaine on isolated rat sciatic nerves is given in Fig. 2. This sample CNAP recording demonstrates that levobupivacaine administration suppresses the CNAPs recorded from isolated nerves in a time-dependent manner, completely abolishing the CNAPs within $25 \mathrm{~min}$. This effect was found to be reversible. Changes in the area under CNAP, CNAP amplitude, conduction velocities of the fastest $\left(\mathrm{CV}_{\text {init }}\right)$ and medium velocity $\left(\mathrm{CV}_{\text {peak }}\right)$ fiber groups were computed using CNAP data at 5-min intervals after the administration $(t=0)$ of levobupivacaine (Fig. 3).

\section{Multiple excitability measurements}

A representative CMAP recording from the gastrocnemius muscle (as in Fig. 1) demonstrating the presence and absence of levobupivacaine is given in Fig. 4. Excitability parameters of the rat sciatic nerve associated with CMAPs are summarized for CON and LEV groups in Table I.

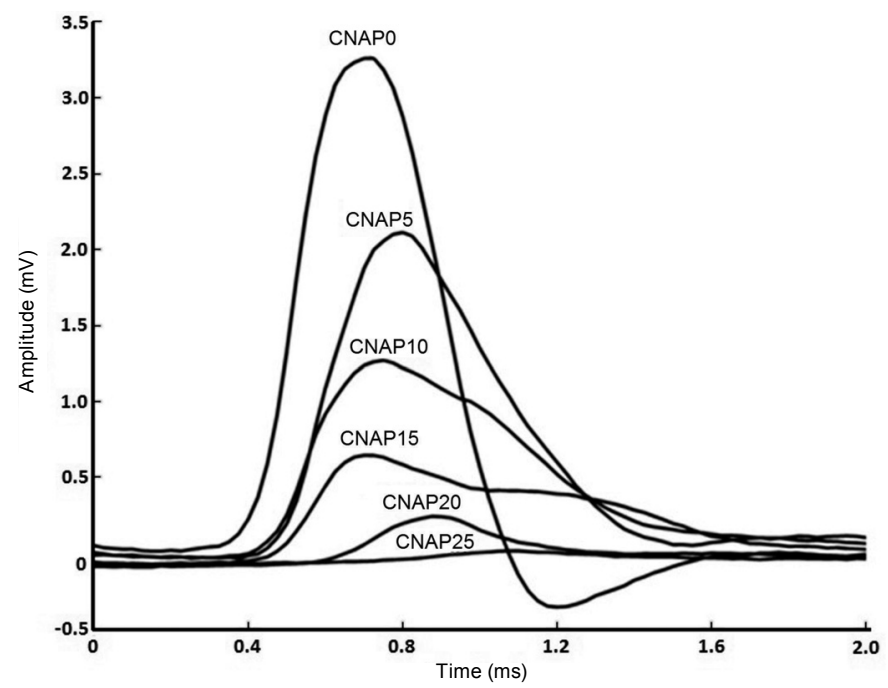

Fig. 2. Representative CNAP samples recorded in vitro from isolated rat sciatic nerves. CNAP curves belong to time sequences starting with the initial recording (CNAP0) and then 5, 10, 15, 20, 25 min (CNAP5, CNAP10, CNAP15, CNAP20 and CNAP25) after $1.7 \mathrm{mmol} \mathrm{L}^{-1}$ levobupivacaine administration. 
a)

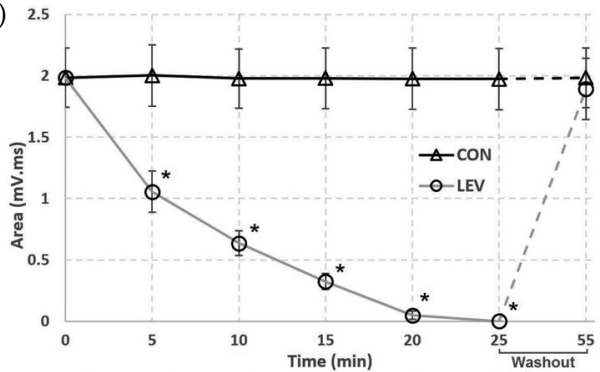

c)

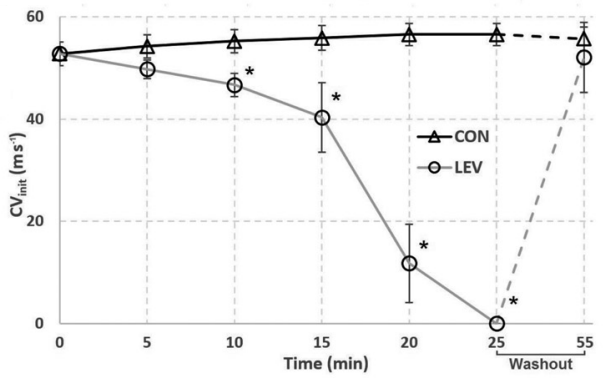

b)

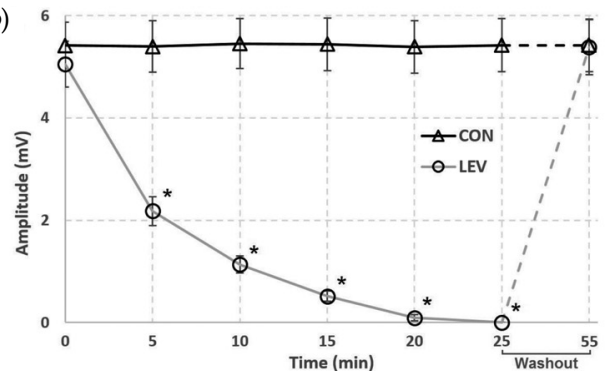

d) 5

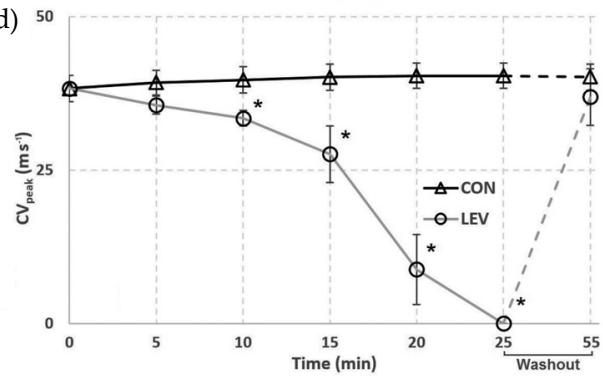

Fig. 3. Time dependent in vitro effect of levobupivacaine $\left(1.7 \mathrm{mmol} \mathrm{L}^{-1}\right)$ and after a 30 -minute washout period on different CNAP parameters: $a$ ) area, b) amplitude, c) conduction velocity of the fastest fibers $\left(\mathrm{CV}_{\text {init }}\right)$ and $\left.\mathrm{d}\right)$ conduction velocity of medium fibers $\left(\mathrm{CV}_{\text {peak }}\right)$. The values are given as mean $\pm \mathrm{SEM}$, $n=8$.

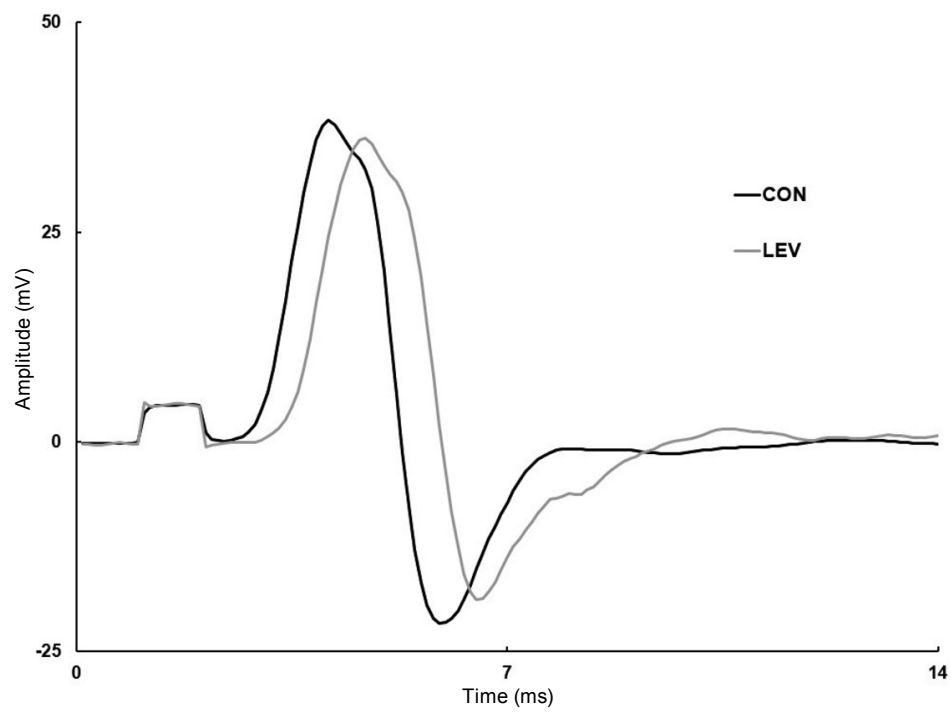

Fig. 4. A representative CMAP recording from the gastrocnemius muscle (as shown in Fig. 1), demonstrating the presence or absence of levobupivacaine. 
Table I. Multiple excitability parameters of the rat sciatic nerve for CON and LEV groups

\begin{tabular}{|c|c|c|}
\hline Excitability measure & $\mathrm{CON}^{\mathrm{a}}$ & $\mathrm{LEV}^{\mathrm{a}}$ \\
\hline \multicolumn{3}{|l|}{ Stimulus-response relationship } \\
\hline Stimulus for $50 \%$ max response $(\mathrm{mA})$ & $2.56 \pm 0.23$ & $3.39 \pm 0.22^{*}$ \\
\hline Peak CMAP (mV) & $34.68 \pm 1.39$ & $34.44 \pm 2.01$ \\
\hline \multicolumn{3}{|l|}{ Strength-duration relationship } \\
\hline Strength-duration time constant (ms) & $0.27 \pm 0.01$ & $0.21 \pm 0.02^{*}$ \\
\hline Rheobase (mA) & $1.94 \pm 0.18$ & $2.70 \pm 0.19^{*}$ \\
\hline Latency (ms) & $3.34 \pm 0.06$ & $3.66 \pm 0.07$ \\
\hline \multicolumn{3}{|l|}{ Threshold electrotonus } \\
\hline $\mathrm{TEd}_{40}(10-20 \mathrm{~ms})$ & $39.92 \pm 0.68$ & $37.58 \pm 2.01$ \\
\hline $\mathrm{TEd}_{40}$ (peak) & $40.21 \pm 0.54$ & $38.85 \pm 1.73$ \\
\hline $\operatorname{TEd}_{40}(90-100 \mathrm{~ms})$ & $28.10 \pm 0.80$ & $25.73 \pm 2.20$ \\
\hline $\mathrm{TEd}_{40}$ (undershoot) & $-11.13 \pm 0.67$ & $-12.59 \pm 1.14$ \\
\hline $\mathrm{TEd}_{20}$ (peak) & $20.78 \pm 0.23$ & $19.57 \pm 0.10$ \\
\hline $\mathrm{TEh}_{40}(10-20 \mathrm{~ms})$ & $-45.53 \pm 0.72$ & $-42.79 \pm 1.79$ \\
\hline $\mathrm{TEh}_{40}(20-40 \mathrm{~ms})$ & $-49.30 \pm 1.22$ & $-45.28 \pm 2.43$ \\
\hline $\mathrm{TEh}_{40}(90-100 \mathrm{~ms})$ & $-50.82 \pm 1.59$ & $-46.92 \pm 2.75$ \\
\hline $\mathrm{TEh}_{40}$ (overshoot) & $4.35 \pm 0.55$ & $6.56 \pm 0.63$ \\
\hline \multicolumn{3}{|l|}{ Current threshold relationship curve } \\
\hline Resting slope of the curve & $1.14 \pm 0.03$ & $1.47 \pm 0.29$ \\
\hline Minimum slope of the curve & $0.39 \pm 0.01$ & $0.38 \pm 0.02$ \\
\hline Hyperpolarizing slope of the curve & $0.39 \pm 0.01$ & $0.38 \pm 0.02$ \\
\hline \multicolumn{3}{|l|}{ Recovery cycle } \\
\hline Refractoriness at $2.5 \mathrm{~ms}(\%)$ & $37.74 \pm 4.30$ & $65.90 \pm 5.20^{\mathrm{b}}$ \\
\hline Peak super-excitability (\%) & $-6.90 \pm 2.96$ & $-6.71 \pm 2.56$ \\
\hline Peak late sub-excitability (\%) & $2.87 \pm 0.50$ & $2.86 \pm 0.91$ \\
\hline
\end{tabular}

a Values are given as mean $\pm \mathrm{SEM}, n=8$.

b Statistically significant difference vs. CON: $\mathrm{p}<0.05$.

Comparison of the stimulus response relation ship of LEV and CON is given in Fig. 5a. A significant increase, compared to $\mathrm{CON}$, was found in stimulus current strength (in $\mathrm{mA}$ ), yielding $50 \%$ of maximal response (Fig. 5b).

Curves associated with strength-duration properties of CON and LEV groups are given in Fig. 6a. According to Weiss's law, as the duration of a test stimulus is increased, current strength required to excite a single nerve fiber or bundle decreases exponentially, eventually flattening out a symptotically for long stimulus durations to reach a value called rheobase (5). The excitability parameters, strength-duration time constant (SDTC) and rheobase values derived from these curves are also given as a bar graph in Figs. $6 \mathrm{~b}$ and 

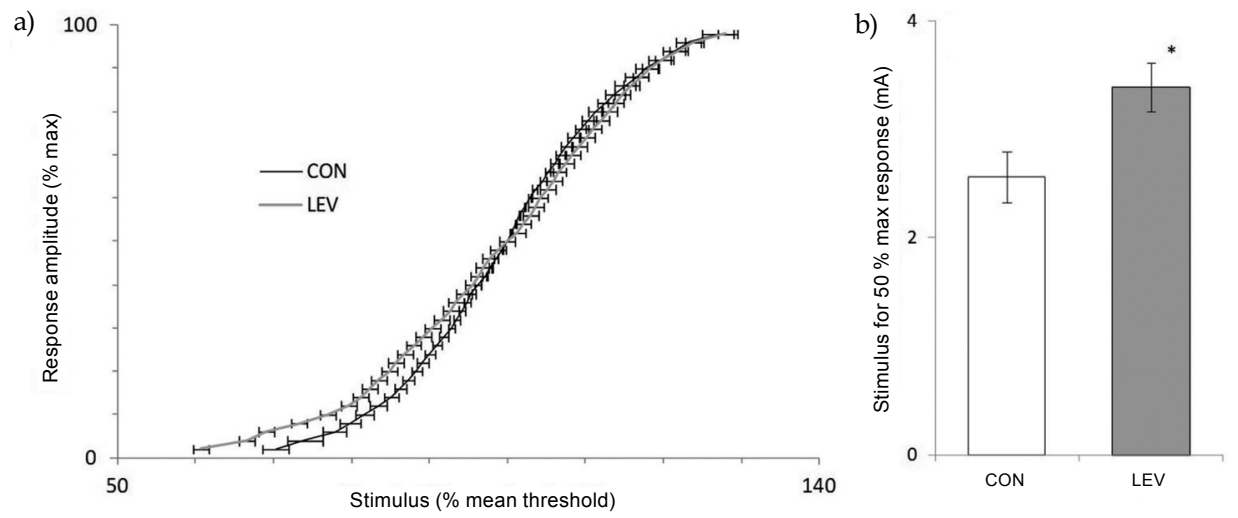

Fig. 5. Stimulus response relationship for CON and LEV groups: a) curves and b) stimuli (mA) for $50 \%$ maximal response (mean \pm SEM, $n=8$, for both groups). Significant difference $v$ s. CON: ${ }^{*} p<0.05$.
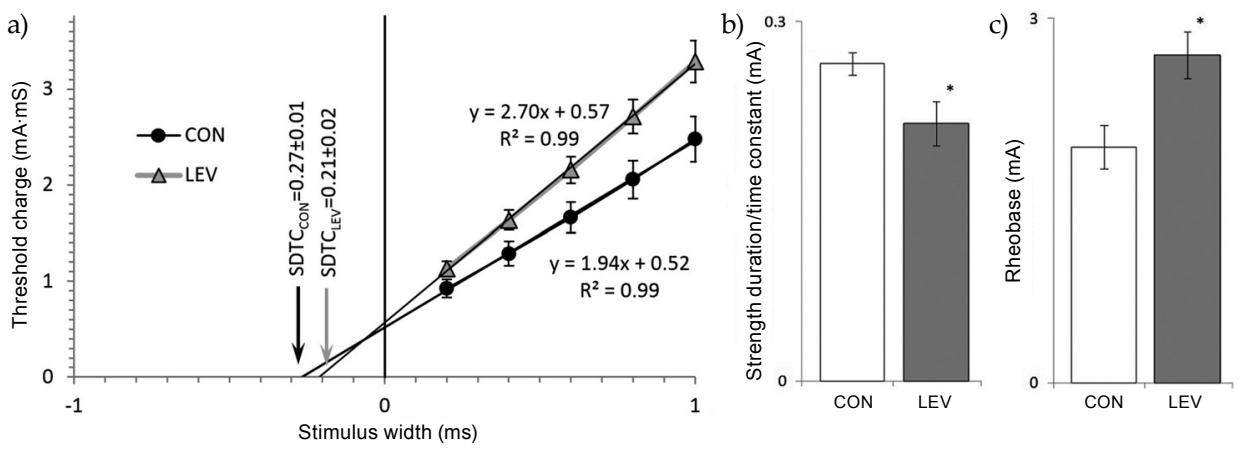

Fig. 6. a) Charge-duration relationship for CON and LEV groups; b) strength-duration time constant (SDTC); c) rheobase values calculated by line fitting (mean \pm SEM, $n=8$ for both groups). Significant difference $v$ s. CON: ${ }^{*} p<0.05$.

6c. SDTC and rheobase values of LEV, derived from these curves, were both found to be significantly different from those for CON (1.94 $\mathrm{mA}$ and $0.28 \mathrm{~ms} v s .2 .7 \mathrm{~mA}$ and $0.22 \mathrm{~ms})$.

The threshold electroton us protocol aims to track the changes in excitability during and after the long-duration depolarization and hyperpolarization of sub-threshold current pulses. Threshold changes are plotted as percent threshold reduction, so that depolarizing responses are plotted upwards, which is more convenient for electrotonus. Mean threshold changes during and after the depolarization and hyperpolarization of sub-threshold current of $100 \mathrm{~ms}$, corresponding to 40 and $20 \%$ of maximal response (i.e., $\mathrm{TEd}_{40}, \mathrm{TEd}_{20}$ and $\mathrm{TEh}_{40}$, $\mathrm{TEh}_{20}$, resp., for depolarizing and hyperpolarizing currents) for CON and LEV groups, are 
given in Fig. 7a. The percent threshold change curve of CON and LEV groups following depolarization revealed no significant difference for either $\mathrm{TEd}_{40}$ or $\mathrm{TEd}_{20}$. Similarly, no significant difference following hyperpolarization was found for either $\mathrm{TEh}_{40} \mathrm{Or}^{\mathrm{T}} \mathrm{Th}_{20}$.

In the current threshold relationship, for an unconditioned test potential, the strength of the conditioning current is varied from +50 to $-100 \%$ of the threshold while keeping its duration constant. One can then obtain the curve for the current threshold relationship by delivering the test pulse of $1 \mathrm{~ms}$ duration at the end of polarizing current, corresponding to the rectification due to $\mathrm{K}^{+}$channels' activation, i.e., the inward rectifier. Current-threshold relationships for depolarization and hyperpolarization for CON and LEV groups were found to be similar (Fig. 7b), without any significant difference.

Recovery cycle $(\mathrm{RC})$ properties, showing the relative refractory period, and providing information about super-excitability and late sub-excitability, are given in Fig. 8a for both CON and LEV. The RC curve for LEV was found to be shifted to the right, meaning that the absolute refractory period for LEV had increased compared to CON ( $\tau_{0}$ in Fig. 8a). The associated average relative refractory period values were found to increase significantly for LEV $(p<0.05)$ compared to CON (Fig. 8b).

\section{In vitro conduction studies}

In the experiments conducted in vitro, isolated rat sciatic nerves manifested a characteristic electrophysiological response to levobupivacaine $\left(1.7 \mathrm{mmol} \mathrm{L}^{-1}\right)$, i.e., a gradual CNAP depression with time. All responses to supramaximal stimuli were found to vanish within 25 min (Figs. 2 and 3). Despite the fact that there is a very limited number of in vitro nerve conduction studies in the literature, this complete blocking effect of levobupivacaine is consistent with a recent study reporting that the maximum effect of levobupivacaine $(0.5$ $\mathrm{mmol} \mathrm{L}^{-1}$ ) on the frog sciatic nerve CNAP amplitude occurred 20 minutes following exposure (14). A similar response is reported in another study of motor and sensory functions

a)

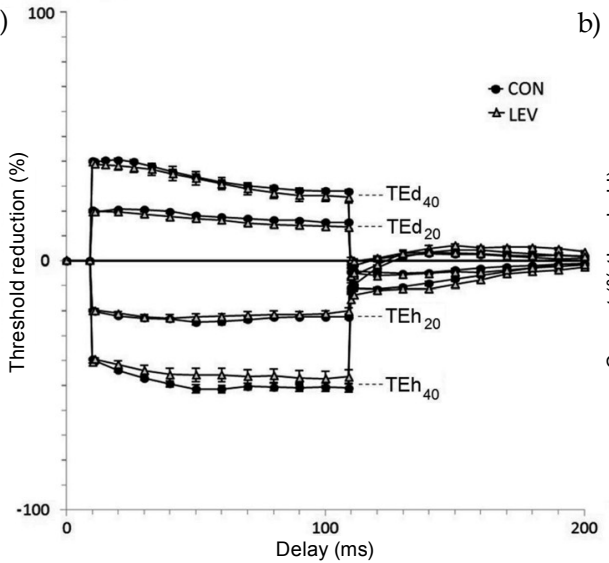

b)

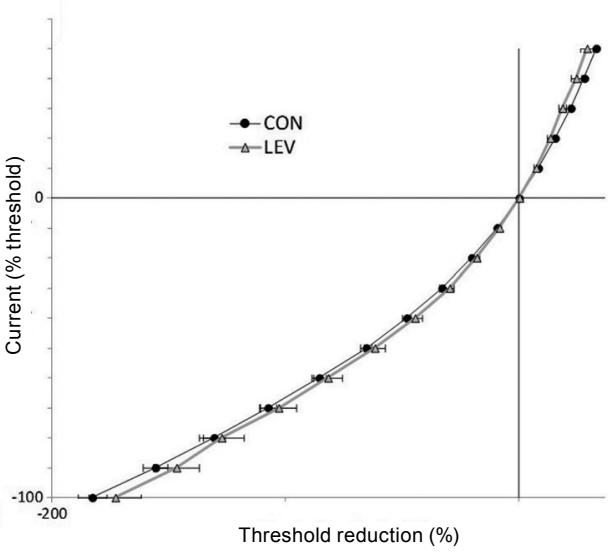

Fig. 7. a) Threshold electrotonus; b) current threshold relationship for CON and LEVgroups (mean \pm SEM, $n=8$ for both groups). 

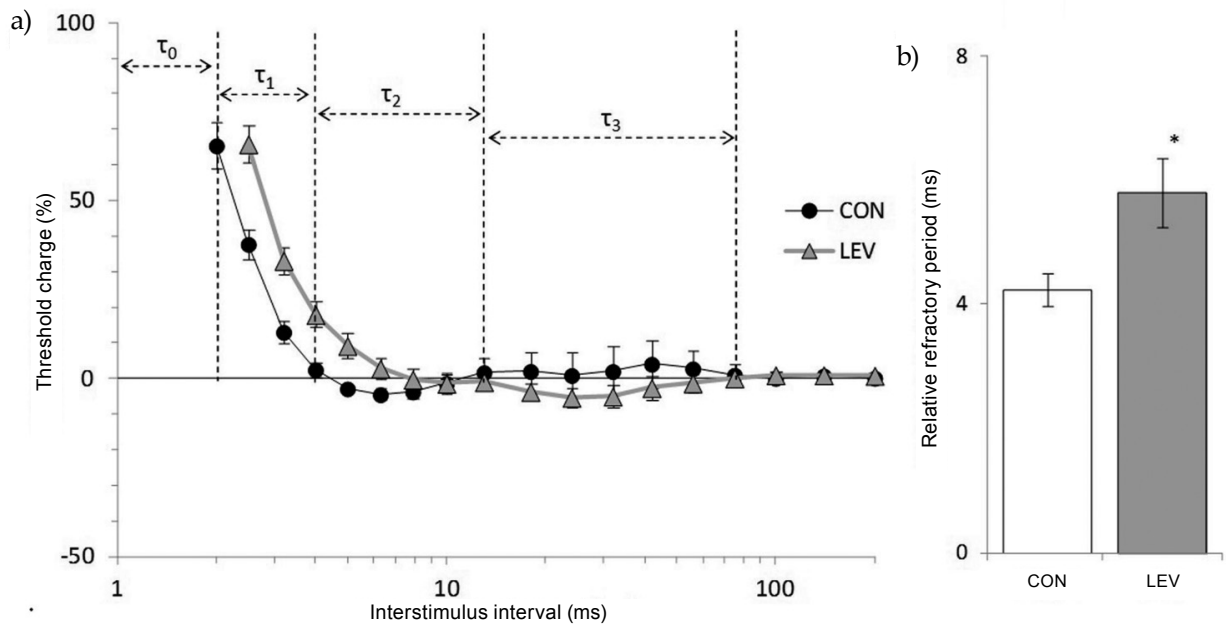

Fig. 8. a) Recovery cycle following the supramaximal conditioning stimulus curves on a logarithmic time-scale; b) average relative refractory period values for CON and LEV groups (mean \pm SEM, $n=8$ ) for both groups). Significant difference vs. CON: ${ }^{*} p<0.05 . \tau_{0}, \tau_{1}, \tau_{2}, \tau_{3}$ correspond to the absolute refractory, relative refractory, super-normal and sub-normal periods, respectively.

in an in vivo behavioral method, however, for a relatively higher dose than we used (15). Despite the difference in doses, considering the location and volume of injections, the impacts due to levobupivacaine could be considered to be the same.

The area of a CNAP waveform can be used as a measure of the number of activated nerve fibers in the nerve trunk (16). The significant area decrease $(42 \%)$ observed in the $5^{\text {th }}$ minute following drug administration (Fig. 3a) can be interpreted as the block of fiber groups having different conduction velocities, based on our previous studies (17). Levobupivacaine was also found to cause a significant decrease $(\sim 56 \%)$ in CNAP amplitude in the $5^{\text {th }}$ minute (Fig. 3b). Although the decrease is quite dramatic, amplitude change may not be a reliable measure of the duration of an anesthetic agent. Due to conduction velocity shifts between fiber groups, there still seem to exist active fibers conducting at a relatively lower velocity. It is known that the peak region of CNAP mainly consists of fiber shaving a relatively lower conduction velocity (18-20) than the fastest group. While the fast fibers, i.e., having a larger diameter and lower threshold, contribute to the initial segment of CNAP, the fibers having are latively slower conduction velocity, i.e., having a smaller diameter and high threshold, contribute to the tail section of the CNAP waveform $(21,22)$.

Conduction velocity changes calculated with respect to the on set $\left(\mathrm{CV}_{\text {init }}\right)$ and peak $\left(\mathrm{CV}_{\text {peak }}\right)$ instants of CNAP (Figs. 3c and 3d) confirmed that there should still exist a certain amount of fibers conducting in the $5^{\text {th }}$ minute after levobupivacaine $\left(0.05 \%, 1.7 \mathrm{mmol} \mathrm{L}^{-1}\right)$ administration. It is also apparent from Figs $3 \mathrm{c}$ and $3 \mathrm{~d}$ that the significant decrease of both $\mathrm{CV}_{\text {init }}$ and $\mathrm{CV}_{\text {peak }}$ starts in the $20^{\text {th }}$ minute. Therefore, the time points at which the dramatic decrease occurs may be considered as a reliable measure for the maximum effect of levobupivacaine. 


\section{In vivo excitability studies}

The time course of a drug effect is an important issue. In the conventional in vitro CNAP recording methods, there are two concerns that should be taken into account: the continuous action of levobupivacaine on the isolated nerve and the amount of impeding forces against diffusion. Therefore, we also tested the time point at which the maximal effect of levobupivacaine occurred by tracking the CMAP amplitude recorded from the gastrocnemius muscle. The CMAP results suggested the maximum effect to occur in the $25^{\text {th }}$ minute (data not shown), which is the same time point yielded by CNAP recordings. Therefore, this time point was chosen as the baseline for testing nerve excitability changes while applying the TT method.

Another finding from the TT method was the stimulus-response curve. As expected, it had a sigmoid shape for both CON and LEV groups (Fig. 5a). Although the stimulating current strength (mA) for $50 \%$ maximal response was significantly different for CON and LEV (Fig. $5 \mathrm{~b}$ and Table I, $p<0.05)$, the associated peak CMAP values $(\mathrm{mV})$ were almost the same. This finding roughly reflects the decrease in excitability due to LEV. However, a more detailed interpretation would be that levobupivacaine reduces the excitability of low-threshold fibers, i.e., faster fiber groups, due to the very fact that it is relatively harder to excite slower fiber groups. Besides, it did not block conduction completely as it was the case of the isolated nerve.

Rheobase and chronaxie (SDTC) provide a conventional measure of nerve excitability and can be calculated using data from the CNAP recording method, which, however, requires multiple recordings. The TT method, on the other hand, makes it possible to determine them easily, as shown by several experimental and clinical studies (5). The effects of levobupivacaine on rheobase and SDTC are given in Fig. 6 and Table I, where a negative correlation was found between the rheobase (Fig. 6c) and SDTC (Fig. 6b). Rheobase and SDTC are nodal properties influenced by the nodal membrane excitability. Their dependence on the voltage level is consistent with persistent $\mathrm{Na}^{+}$channels' $\left(\mathrm{Na}_{\mathrm{p}}\right)$ behavior. $\mathrm{Na}_{\mathrm{p}}$ channels are active near the threshold and their inactivation is not as rapid as that of transient $\mathrm{Na}^{+}$channels $(6,7,23)$. Depolarization decreases due to the $\mathrm{Na}^{+}$current through $\mathrm{Na}_{\mathrm{p}}$ resulting in lower rheobase, whereas hyperpolarization has the opposite effect. As the charge-duration relationship suggests, SDTC takes values in the opposite direction from the rheobase (6). Hence, the membrane is prevented from hyperpolarizing due to leakage of $\mathrm{Na}^{+}$through $\mathrm{Na}_{\mathrm{p}}$ channels, contributing to $3-5 \%$ of the total $\mathrm{Na}^{+}$channel activity during the resting state of the membrane.

A decrease in membrane potential or a change in passive membrane properties would cause a decrease in SDTC values. Since levobupivacaine decreases $\mathrm{Na}^{+}$channels' permeability, the membrane should become hyperpolarized. This in turn leads to a decrease in SDTC, which is a strong evidence for a decrease in membrane excitability. This result suggests that proper functioning of persistent $\mathrm{Na}^{+}$channels in the rat sciatic nerve is restricted within 25 min after levobupivacaine injection, which may be responsible for decreased excitability.

Threshold electrotonus is another protocol enabling us to monitor the inter-nodal conductance, which in turn yields a characteristic pattern of the change in excitability during and after long-term depolarizing and hyperpolarizing currents unable to trigger an action potential. Under such conditions, the strength of the test pulse required to obtain 
the predetermined target CMAP amplitude is also changed relative to the pulse onset. In our study, target amplitudes were chosen to yield 20 and $40 \%$ of CMAP maximum amplitude. The rationale behind choosing two different target response amplitudes is that the most excitable fiber groups are the fastest ones and may result in $20 \%$ target response, where the rest are the medium ones responsible for $40 \%$ target response.

Threshold electrotonus curves revealed no difference for either TE $\mathrm{d}_{40}$ or $T E \mathrm{~d}_{20}$. The patterns followed by the $\mathrm{TEh}_{40}$ and $\mathrm{TEh}_{20}$ curves for both CON and LEV were almost the same (Fig. 7a). In the $\mathrm{TEd}_{40}$ curve, excitability remained almost the same over the first $20 \mathrm{ms,}$ with a slight decrease up to $100 \mathrm{~ms}$ TEd ${ }_{20}$ curves of CON and LEV followed the same trend. These findings are coherent with the TE (\%) measures given in Table I. Since a change in nerve excitability during TEd is controlled successively by passive membrane properties (i.e., resistance and capacitance) and also via fast and slow $\mathrm{K}^{+}$channel conductance $(5,6)$, the threshold electrotonus findings can be interpreted in such a way that levobupivacaine has no significant effect on the passive membrane properties and on $\mathrm{K}^{+}$conductance for either fastest or medium velocity fiber groups. Besides, the current-threshold curves for both groups were also found to follow the same trend (Fig. $7 \mathrm{~b}$ ). These results support our suggestion of a limited effect of levobupivacaine on $\mathrm{K}^{+}$channels, since hyperpolarization deactivates inter-nodal $\mathrm{K}^{+}$channels while activating the inward rectifier $\mathrm{K}^{+}$channels (5).

The sequence of change in excitability, following nerve fiber depolarization, is called the recovery cycle, which can be either in an increasing (i.e., below baseline) or decreasing phase (i.e., above baseline). These phases are labeled in Fig. $8 \mathrm{a}$ as $\tau_{0}, \tau_{1}, \tau_{2}$, and $\tau_{3}$ for the CON group. There was a complete rightward shift in the average curve of LEV groups, compared to CON groups, meaning that levobupivacaine influenced the inactivation and deactivation phases of ion channels. Increase in phase $\tau_{0}$ (absolute refractory period) of the LEV group shows that even when the strongest stimuli are applied, the fibers are absolutely unexcitable. This prolongation in $\tau_{0}$ is due to the delay in inactivation of the transient voltage dependent $\mathrm{Na}^{+}$channels. In phase $\tau_{1}$ (relative refractory period), axonal excitability in the LEV group is low, and there is also a significant $(p<0.05)$ extension in duration (Fig. $8 \mathrm{~b}$ ) that may be attributed to the delay caused by levobupivacaine as it causes gradual recovery of transient $\mathrm{Na}^{+}$channels. Axonal excitability increases during phase $\tau_{2}$ (supernormal period). Compared to the $\mathrm{CON}$ group, there is a substantial rightward shift in the LEV group, and its duration is longer. Finally, for phase $\tau_{3}$, the LEV group either vanishes completely or phase $\tau_{2}$ (supernormal period) is delayed towards $\tau_{3}$ as a result of complete shift.

\section{CONCLUSIONS}

The current study was designed to investigate, using two well-established methods, how the long-acting amide type local anesthetic levobupivacaine affects nerve conduction and excitability parameters.

Conventional methods based on the compound nerve action potential recording still keep their place in investigating the state of conduction and fiber diameter distribution in peripheral nerve studies. As it was pointed out before, the time course of the effect of an administered anesthetic drug may be traced precisely by this method. The method also facilitates the estimation of conduction velocity shifts in fiber groups and the excitability 
state of the nerve trunk. Various protocols of the TT method, however, make in vivo investigation of channel kinetics possible, even in humans.

Consequently, this study was aimed to investigate the electrophysiological parameters upon administration of levobupivacaine on isolated sciatic nerves for some time. This study employed an automated TT system to investigate multiple excitability parameters of the rat sciatic nerve, which corresponds to the maximum response following peri-neural injection of levobupivacaine.

Finally, the study has demonstrated that levobupivacaine $\left(0.05 \%, 1.7 \mathrm{mmol} \mathrm{L}^{-1}\right) \mathrm{de}^{-}$ presses conduction in the isolated sciatic nerve in a time-dependent manner, and that complete conduction block occurs 20 minutes after administration. Levobupivacaine causes a decrease in $\mathrm{Na}^{+}$channel activity (or permeability), with an increase in the rheobase and in absolute/relative refractory periods and a decrease of SDTC values, as revealed by the in vivo TT method results. Changes in the assessed parameters could be accepted asevidencefor the decrease in the excitability state of nerve fibers in response to levobupivacaine application.

Acronyms, symbols. - AMPA - $\alpha$-amino-3-hydroxy-5-methyl-4-izoxazolepropionic acid; ARRIVE - Animal research: Reporting of in vivo experiments; CMAP - compound muscle action potential; $\mathrm{CNAP}$ - compound nerve action potential; $\mathrm{CV}_{\text {init }}$ - conduction velocity derived using latency1; $\mathrm{CV}_{\text {peak }}$ - conduction velocity derived using latency 2; GTP - guanosine triphosphate; KONUDAM - N. E. University Experimental Medicine and Application Center; KSPS - kilosamples s ${ }^{-1}$; NMDA $-N$-methyl-D-aspartate; $\mathrm{Na}_{\mathrm{p}}$ - persistent $\mathrm{Na}^{+}$channels; QTRACS - software for applying multiple excitability protocol; QTRACP - software for analyzing multiple excitability data; RETICAP - open-source compound nerve action potential recording software; RC - recovery cycle; SDTC - strength-duration time constant; $\Delta t_{\text {onset }}$ - latency1, time delay between the instant of stimulus delivery and the associated CNAP onset; $\Delta t_{\text {peak }}$ - latency2, time delay between the stimulus delivery instant and the associated CNAP reaching its maximum value; TE - threshold electrotonus; TEd40 - depolarizing sub-threshold current for $40 \%$ of maximal response; TEd 20 - depolarizing sub-threshold current for $20 \%$ of maximal response; TEh 40 - hyperpolarizing sub-threshold current for $40 \%$ of maximal response; TEh20 - hyperpolarizing sub-threshold current for $20 \%$ of maximal response; TRONDNF - multiple excitability protocol; TRPV1 - transient receptor potential vanilloid 1; TT - threshold tracking

\section{REFERENCES}

1. R. H. Foster and A. Markham, Levobupivacaine: a review of its pharmacology and use as a local anaesthetic, Drugs 59 (2000) 551-579; DOI: 10.2165/00003495-200059030-00013.

2. F. Yanagidate and G. R. Strichartz, Local anesthetics, Handb. Exp. Pharmacol. 177 (2006) 95-127.

3. L. J. Dorfman, The distribution of conduction velocity (DCV) in peripheral nerves: a review, Muscle Nerve 7 (1984) 2-11; DOI: 10.1002/mus.880070103.

4. F. Pehlivan, N. Dalkilic and E. Kiziltan, Does the conduction velocity change along the nerve?, Med. Eng. Phys. 26 (2004) 395-401; DOI: 10.1016/j.medengphy.2004.02.009.

5. H. Bostock, K. Cikurel and D. Burke, Review article: Threshold tracking techniques in the study of human peripheral nerve, Muscle Nerve 21 (1998) 137-158; DOI: 10.1002/(SICI)1097-4598 (199802)21:2<137::AID-MUS1>3.0.CO; 2-C.

6. H. Nodera and R. Kaji, Invited review article: Nerve excitability testing and its clinical application to neuromuscular diseases, Clin. Neurophysiol. 117 (2006) 1902-1916; DOI: 10.1016/j.clinph.2006.01.018.

7. A. V. Krishnan, S.S.-Y. Lin, S. B. Park and M. C. Kiernan, Axonal ion channels from bench to bedside: A translational neuroscience perspective, Prog. Neurobiol. 89 (2009) 288-313; DOI: 10.1016/j. pneurobio.2009.08.002. 
8. I. Kara, S. Apiliogullari, S. Bagcı Taylan, H. Bariskaner and J. B. Celik, The effects of dexketoprofen on duration of analgesia to a thermal stimulus when compared with a systemic control in a rat sciatic nerve block with levobupivacaine, Fundam. Clin. Pharmacol. 28 (2014) 205-210; DOI: 10.1111/ fcp.12010.

9. M. C. Kiernan, C. S. Y. Lin, K. V. Andersen, N. M. F. Muray and H. Bostock, Clinical evaluation of excitability measures in sensory nerve, Muscle Nerve 24 (2001) 883-892; DOI: 10.1002/mus.1085.

10. Q. Yang, R. Kaji, N. Hirota, Y. Kojima, T. Takagi, N. Kohara, J. Kimura, H. Shibasaki and H. Bostock, Effect of maturation on nerve excitability in an experimental model of threshold electrotonus, MuscleNerve 23 (2000) 498-506; DOI:10.1002/(SICI)1097-4598(200004)23:4<498::AID-MUS7>3.0.CO;2-U.

11. A. George and H. Bostock, Multiple measures of axonal excitability in peripheral sensory nerves: An in vivo rat model, Muscle Nerve 36 (2007) 628-636; DOI: 10.1002/mus.20851.

12. D. Boerio, L. Greensmith and H. Bostock, Excitability properties of motor axons in the maturing mouse, J. Peripher. Nerv. Syst. 14 (2009) 45-53; DOI: 10.1111/j.1529-8027.2009.00205.x.

13. J. Cohen, Statistical Power Analysis for the Behavioral Sciences, Lawrence Earlbaum Associates, Hillsdale (NJ) 1988, pp. 20-26.

14. Y. Uemura, T. Fujita, S. Ohtsubo, N. Hirakawa, Y. Sakaguchi and E. Kumamoto, Effects of various antiepileptics used to alleviate neuropathic pain on compound action potential in frog sciatic nerves: comparison with those of local anesthetics, Biomed. Res. Int. 2014 (2014) 540238; DOI: $10.1155 / 2014 / 540238$.

15. C. J. Sinnott and G. R. Strichartz, Levobupivacaine versus ropivacaine for sciatic nerve block in the rat, Reg.Anesth. Pain Med. 28 (2003) 294-303; DOI: 10.1016/S1098-7339 (03)00188-3.

16. H. Bariskaner, M. Ayaz, F. B. Guney, N. Dalkilic and O. Guney, Bupivacaine and ropivacaine: comparative effects on nerve conduction block, Methods Find. Exp. Clin. Pharmacol. 29 (2007) 337341; DOI: 10.1358/mf.2007.29.5.1117558.

17. N. Dalkilic, S. Tuncer, H. Bariskaner and E. Kiziltan, Effect of tramadol on the rat sciatic nerve conduction: anumerical analysis and conduction velocity distribution study, Yakugaku Zasshi $\mathbf{1 2 9}$ (2009) 485-493; DOI: 10.5505/agri.2011.72792.

18. R. S. W. Wijesinghe, F. L. Gielen and J. P. Wikswo, A model for compound action potentials and currents in a nerve bundle I: The forward calculation, Ann. Biomed. Eng. 19 (1991) 43-72; DOI: 10.1007/BF02368460.

19. G. Hirose, Y. Tsuchitani and J. Huang, A new method for estimation of nerve conduction velocity distribution in the frequency domain, Electroencephalogr. Clin. Neurophysiol.63 (1986) 192-202; DOI: 10.1016/0013-4694 (86)90013-1.

20. N. Dalkilic and F. Pehlivan, Comparison of fiber diameter distribution deduced by modelling compound action potentials recorded by extracellular and suction techniques, Int. J. Neurosci. 122 (2002) 913-930; DOI: 10.1080/00207450290025923.

21. G. Schalow and G. A. Zach, Nerve compound action potentials analysed with the simultaneously measured single fibre action potentials in humans, Electromyogr. Clin. Neurophsiol. 34 (1994) 451-465.

22. S. Tuncer, N. Dalkilic, H. H. Esen and M. C. Avunduk, An early diagnostic tool for diabetic neuropathy: Conduction velocity distribution, Muscle Nerve 43 (2010) 237-244; DOI: 10.1002/mus. 21837.

23. H. G. Ferreira and M. W. Marshall, The Biophysical Basis of Excitability, Cambridge Univ. Press, Cambridge 1985, pp. 8-194. 